\title{
1 Background selection under evolving 2 recombination rates
}

3 Tom R. Booker ${ }^{1, *}$, Bret A. Payseur ${ }^{2}$ and Anna Tigano ${ }^{3}$

4 1. Department of Zoology, University of British Columbia, Vancouver Campus, Vancouver, 5 BC, Canada

6 2. Laboratory of Genetics, University of Wisconsin - Madison, Madison, WI, USA.

3. Department of Biology, University of British Columbia, Okanagan Campus, Kelowna, BC, Canada

$9 \quad *$ Correspondence: booker@zoology.ubc.ca 


\section{Abstract}

11 Background selection (BGS), the effect that purifying selection exerts on sites linked to

12 deleterious alleles, is expected to be ubiquitous across eukaryotic genomes. The effects of BGS

13 reflect the interplay of the rates and fitness effects of deleterious mutations with

14 recombination. A fundamental assumption of BGS models is that recombination rates are

15 invariant over time. However, in some lineages recombination rates evolve rapidly, violating

16 this central assumption. Here, we investigate how recombination rate evolution affects genetic

17 variation under BGS. We show that recombination rate evolution modifies the effects of BGS in

18 a manner similar to a localised change in the effective population size, potentially leading to an

19 underestimation of the genome-wide effects of selection. Furthermore, we find evidence that

20 recombination rate evolution in the ancestors of modern house mice may have impacted

21 inferences of the genome-wide effects of selection in that species. 


\section{Introduction}

23 Different modes of selection (e.g. positive, purifying and balancing) all affect genetic variation

24 at sites linked to the actual targets of selection (reviewed in Charlesworth 2009). In the case of

25 purifying selection, the removal of deleterious mutations causes linked neutral variants to be

26 lost along with them through a process referred to as background selection (BGS; Charlesworth

27 et al. 1993). Of the mutations that affect fitness in natural populations, the vast majority are

28 likely deleterious with a comparatively small proportion of beneficial mutations (Eyre-Walker

29 and Keightley 2007). For those reasons, it has been proposed that BGS is ubiquitous across

30 eukaryotic genomes and should be incorporated into null models for population genomics

31 (Comeron 2017; Johri et al. 2020). Indeed, recent studies have used BGS to set baseline

32 patterns for identifying the locations and effects of positively selected mutations (DeGiorgio et

33 al. 2016; Campos et al. 2017) and understanding Lewontin's paradox of genetic diversity

34 (Buffalo 2021). Interpreting genome-wide patterns of genetic diversity in terms of BGS,

35 however, requires accurate estimates of population genetic parameters, particularly

36 recombination rates.

37 In many species, the recombination rate per base pair $(r)$ varies across the genome both

38 between and within chromosomes (Stapley et al. 2017). For example, in the house mouse (Mus

39 musculus) the average $r$ for chromosome 19 (the shortest chromosome) is around 60\% higher

40 than for chromosome 1 (the longest chromosome)(Cox et al. 2009). The requirement of at least

41 one cross-over per chromosome per meiosis in mammals causes shorter chromosomes to

42 recombine at a higher average rate than longer ones (Pardo-Manuel et al. 2001; Segura et al. 
43 2013; Dumont 2017). Local recombination rates can vary substantially across chromosomes as

44 well and in some cases this variation is predicted by gross features of chromosome architecture

45 such as the locations of centromeres and telomeres (Paigen et al. 2008). Actual recombination

46 events in mice are typically restricted to narrow windows of the genome (on the order of 1-5

$47 \mathrm{Kbp}$ ), referred to as hotspots (Paigen et al. 2008). The positions of recombination hotspots in

48 mice, and in some other vertebrates, are determined by the binding of a protein encoded by

49 the PRDM9 gene to specific DNA motifs (Baudat et al. 2010; Baker et al. 2017), although

50 hotspots are still observed in PRDM9 knockout lines and dogs, which lack a functional copy of

51 PRDM9 (Brick et al. 2012; Auton et al. 2013).

52 Estimates of $r$ can be obtained empirically by examining the inheritance of genetic markers

53 through controlled crosses or through pedigrees, or by comparing an individual's genome to

54 that of its gametes (e.g. Sun et al. 2019). Both methods reconstruct recombination events over

55 one or a few generations, and thus provide estimates of $r$ for contemporary populations.

56 Alternatively, estimates of $r$ can be obtained indirectly by analysing patterns of linkage

57 disequilibrium across the genome (e.g. Spence and Song 2019), in which case estimates reflect

58 both recent and ancestral recombination events. Whether recombination rates are estimated

59 from marker transmission or population genetics, using such estimates when analysing of

60 variation across the genome in terms of BGS implicitly assumes that the recombination

61 landscape has not changed over the time in which patterns of diversity have been established.

62 However, recombination rate landscapes can evolve very rapidly in some lineages. For example,

63 due to the relationship between chromosome size and average $r$, changes in chromosome

64 length (i.e. karyotype evolution) may induce changes in $r$. The lineage leading to Mus musculus 
$65(2 n=40)$ has experienced large chromosomal rearrangements since it shared a common

66 ancestor with Mus pahari $(2 n=48)$ 3-5 million years ago (Thybert et al. 2018). Moreover,

67 different populations of Mus musculus domesticus harbouring different karyotypes exhibit

68 different genomic landscapes of recombination (Vara et al. 2021). Chromosomal fusions can

69 exhibit meiotic drive (Chmátal et al. 2014) so new karyotypes may spread to fixation very

70 rapidly. Even mice with the same karyotype vary in regional recombination rate across

71 substantial proportions of the genome (Dumont et al. 2011; Wang et al 2017) and in total

72 number of crossovers (Dumont and Payseur 2011; Peterson and Payseur 2021), both within and

73 between subspecies. There is also evidence that $P R D M 9$, the gene that encodes the protein

74 that dictates the locations of recombination events, has undergone recurrent bouts of positive

75 selection in mice (Oliver et al. 2009) and natural populations of M. musculus spp. possess

76 various PRDM9 alleles corresponding to different suites of recombination hotspots (Smagulova

77 et al. 2016). Overall, there is clear evidence from mice that recombination rates can evolve on

78 broad and fine scales.

79 Changes in the recombination rate over time may influence patterns of genetic variation across

80 the genome (Comeron 2017). For example, chromosomal fusions would decrease

81 recombination rates experienced by individual nucleotides in the fused chromosomes, and thus

82 increase the effects of BGS and other processes mediated by recombination. Consistent with

83 this, Cicconardi et al. (2021) found evidence suggesting that chromosomes that underwent

84 fusions in the ancestors of extant Heliconius butterfly species now exhibit reduced

85 recombination rates and $\pi$ presumably due to amplified BGS effects. Following evolution of the

86 recombination rate landscape there will be a lag period wherein patterns of genetic variation 
87 more closely reflect ancestral recombination rates than derived rates. Over time, as new

88 deleterious mutations arise and cause BGS, patterns of genetic variation will come to reflect

89 derived recombination rates. Depending on the extent and rate of recombination rate

90 evolution, population genomic analysis of lineages that are still within the lag period may be

91 obscured. In this paper, we examine how patterns of neutral genetic variation under BGS

92 respond to evolution of the recombination rate and describe how this could affect and have

93 affected analyses that are used to identify the effects of selection on a genome-wide scale. 


\section{Results}

\section{Background selection under evolving recombination rates}

96 The effects of BGS reflect the interplay of purifying selection and recombination (Nordborg et

97 al. 1996), so changes to the recombination rate will influence the effects of BGS. An increase in

98 the recombination rate between neutral sites and sites subject to purifying selection will

99 decrease the effect of BGS and vice versa for a decrease in the recombination rate. At a neutral

100 locus $v$, coalescence times under BGS $\left(T_{B G S, v}\right)$ are shorter than those expected under neutrality

$101\left(T_{\text {Neutral }}\right)$ (Nordborg et al. 1996) and the effect of BGS is often expressed as $B_{v}=$

$102 T_{B G S, v} / T_{\text {Neutral }}$ (e.g. Nordborg et al. 1996). Consider a population that underwent a change in

103 the recombination rate such that $v$ experiences a BGS effect of $B_{v}^{\prime}$ under the derived

104 recombination rate regime. Even with instantaneous changes in the recombination rate,

105 genetic variation at $v$ would not reflect $B_{v}^{\prime}$ immediately, as there would be a lag period after

106 recombination rate change wherein coalescence times (and patterns of genetic variation)

107 would more closely reflect $B_{v}$.

108 Under strong purifying selection, BGS resembles a localised reduction in the effective

109 population size, so the period of lag after a change in the recombination rate may resemble the

110 change in coalescence times following a change in the population size. If the recombination

111 rate changed at time $t$ in the past (measured in $2 N_{e}$ generations), then BGS under the new

112 recombination rate can described with:

113

$$
B_{v, t}=B_{v}\left(1+\left(\frac{B_{v}}{B_{v}^{\prime}}-1\right) e^{-t}\right)
$$


114 We obtained Equation 1 by modifying an expression that describes coalescence times after an

115 instantaneous change in the population size from Johri et al. (2020). Note that Pool and Nielsen

116 (2009) provided similar expressions to those given by Johri et al. (2020).

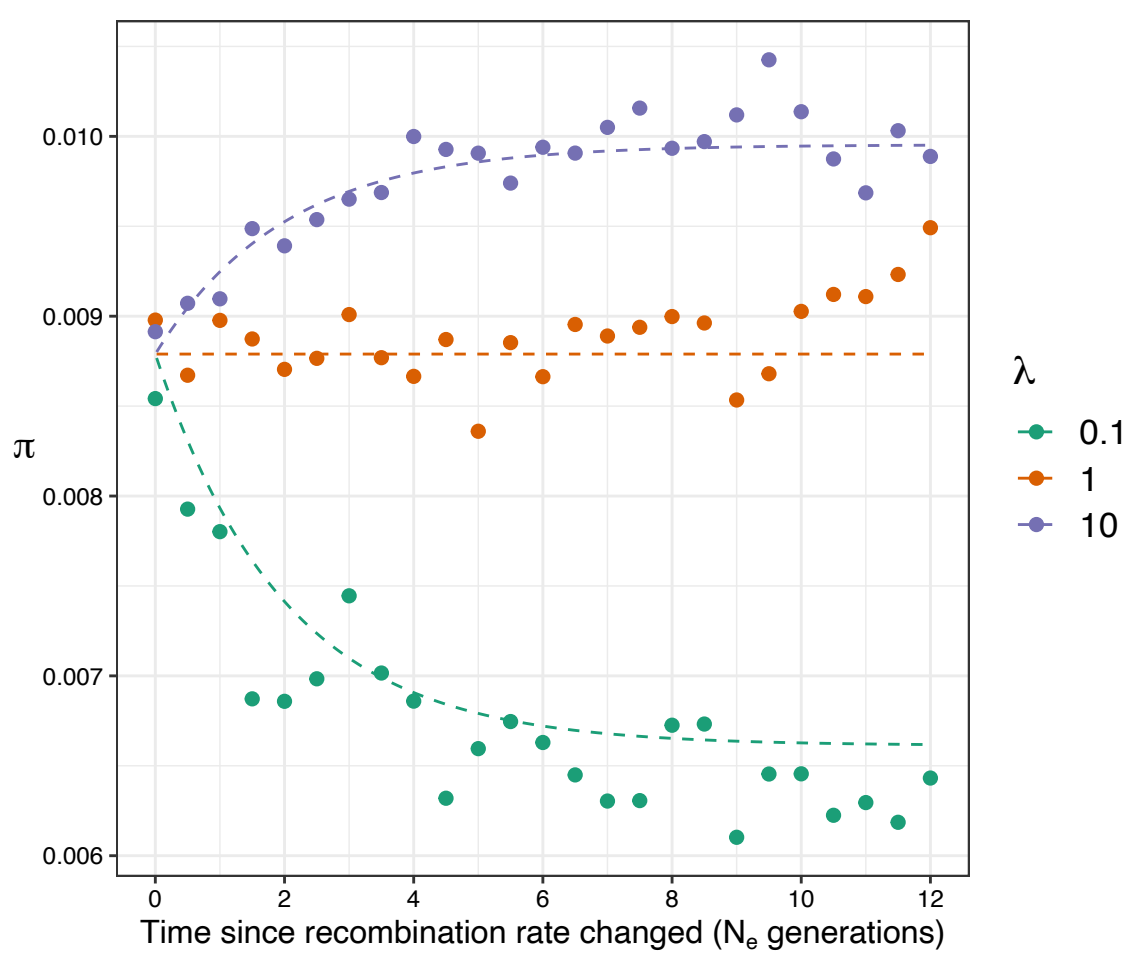

118 Figure 1. The effect of background selection on nucleotide diversity $(\pi)$ over time after 119 recombination rates change by a factor $\lambda$. The dashed lines were calculated using Equation 1

120 and formulae from Nordborg et al (1996). Points indicate the mean from 100 replicate 121 simulations. Nucleotide diversity was calculated for neutral sites 10,000bp away from sites 122 subject to purifying selection.

123 We modelled deleterious mutations occurring in a single functional element (e.g. a protein

124 coding exon) and examined $\pi$ for neutral mutations in and around this region after an

125 instantaeous change in the recombination rate (Figure S1). $\pi$ gradually departs from the

126 expectations based on the ancestral recombination rate over 4Ne generations, when it finally

127 aligns to the derived recombination rate (Figure $1, S 2$ ). Up to $\sim 2 N_{e}$ generations after a change in

128 the recombination rate, $\pi$ more closely resembled the expectation under the ancestral 
129 recombination rate than it did the derived rate (Figure $1, \mathrm{~S} 2$ ). After around $4 N_{e}$ generations,

130 coalescence times closely reflected those expected under BGS given the derived recombination

131 rate, as measured by $\pi$ (Figure 1, S2). When deleterious mutations have nearly neutral

132 deleterious effects, Equation 1 may not predict changes in nucleotide diversity particularly well

133 because in such cases BGS does not resemble a simple reduction in $N_{e}$ (Good et al. 2014;

134 Cvijović et al. 2018).

135 In the case of a population that has recently undergone shifts in the recombination rate

136 landscape (i.e. less than $2 N_{e}$ generations ago), estimates of $r$ from such a population would

137 likely reflect contemporary recombination rates regardless of how they were obtained.

138 Estimates of $r$ from patterns of marker inheritance in crosses or pedigrees always reflect

139 contemporary rates and population genetic estimates (i.e. obtained from patterns of LD) can

140 reflect contemporary recombination rates within $0.5 N_{e}$ generations of a change in $r$ (Figure S3).

141 Depending on the extent and nature of recombination rate evolution, population genomic

142 analyses that compare features of genetic variation to estimates of $r$ could lead to an

143 underestimation of the effects of BGS (and other forms of selection) on patterns of genetic

144 variation.

145 Patterns of genetic variation after evolution of the recombination

146 landscape

147 To demonstrate how population genomic analyses may be affected by changes in $r$, we

148 simulated two scenarios of BGS under evolving recombination rates. In the first, the broadscale 
149 landscape of $r$ was rearranged (Figure S4A). In the second, the locations of recombination

150 hotspots were shifted, as if a new PRDM9 allele had fixed in a population (Figure S4B). In both

151 scenarios, deleterious mutations occurred at random across the genome generating

152 widespread BGS such that there was a positive correlation between $\pi$ and $r$ at equilibrium

153 (Figure 2). For the sake of our analyses have assumed that recombination rate is invariant

154 among individuals, even as heritable variation in recombination rates has been reported in

155 several species (reviewed in Stapley et al. 2017).

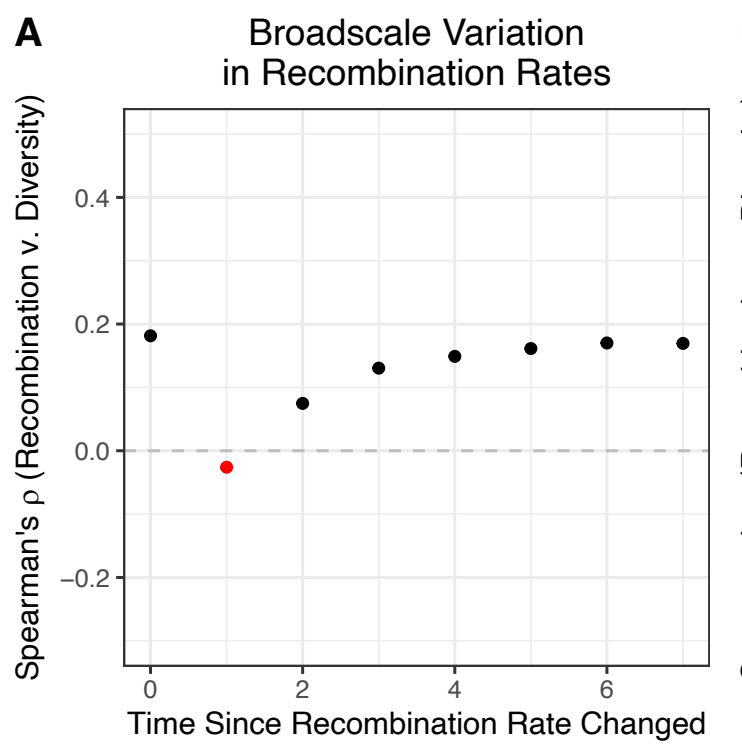

156

157

158

159

160

161

162

163

164

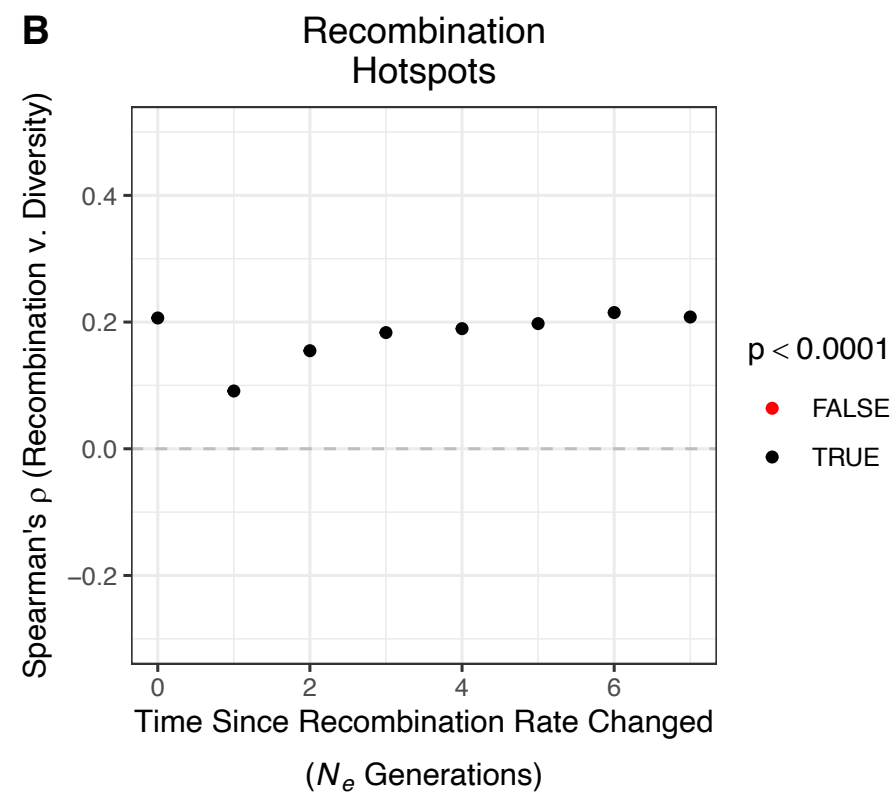

Figure 2. Spearman's correlation between nucleotide diversity $(\pi)$ and recombination rate $(r)$ over time after recombination rates evolve. Panel A shows results for a broad-scale shift in the recombination landscape and panel $B$ shows results for recombination rate evolution by the movement of hotspots. Results are shown for $10 \mathrm{Kbp}$ analysis windows.

A positive correlation between $\pi$ and $r$ is a hallmark of widespread selection across a genome

(Cutter and Payseur 2013), but evolution of the recombination rate may obscure this pattern. In both the scenarios we simulated, changes in $r$ did not influence the average nucleotide diversity across simulated chromosomes (Figure S5), because under the models of recombination rate 
165 evolution we implemented the average map length was constant over time. However, before

166 the change in the recombination rate, there was a positive correlation between $\pi$ and $r$ in both

167 scenarios that was detectable when examining $10 \mathrm{Kbp}, 100 \mathrm{Kbp}$ and $1 \mathrm{Mbp}$ analysis windows

168 (Figure 2,S6). Following changes in the recombination rate landscape under the model of

169 broadscale recombination rate variation, the correlation between $\pi$ and $r$ was either absent or

170 misleading (Figure 2A, S6A). Under the model of recombination hotspot evolution, the

171 correlation between $\pi$ and $r$ was weakened by change in the landscape of hotspots (Figure 2B).

172 In both cases we simulated, a positive correlation between $\pi$ and derived $r$ was restored to

173 levels similar to what had been observed before the recombination maps changed after about

$1744 N_{e}$ generations (Figure 2, S6). Figure 2 shows results for $10,000 \mathrm{bp}$ analysis windows, but

175 similar results were found when examining larger windows (Figure S5).

\section{Rapid recombination rate evolution in house mice}

177 Rapid evolution of recombination rates in Mus musculus may have influenced our ability to

178 identify the effects of selection across that species' genome. Kartje et al. (2020) recently

179 demonstrated that natural populations of M. m. domesticus exhibit a very weak correlation

180 between $\pi$ and $r$ (when examining analysis windows of various widths) and concluded that

181 selection at linked sites exerted only modest effects on genetic variation throughout the

182 genome. This is notable because wild mice are thought to have large effective population sizes

183 for mammals (Leffler et al. 2012) and genome-wide effect of selection is thought to be more

184 pronounced in species with large $N_{e}$ (Cutter and Payseur 2013). As discussed in the

185 Introduction, there is evidence that mice have undergone rapid evolution of the recombination 
186 rate. For example, around 3-5 MYA the lineage leading to $M$. musculus experienced a burst of

187 karyotype evolution (Thybert et al. 2018). If that burst of karyotype evolution affected

188 recombination rates and ancestral mouse populations were very large, then contemporary

189 mice may still be within the lag period described by Equation 1. Patterns of genetic diversity in

190 mice may still be adjusting to historical changes in the recombination rate, and we may see a

191 stronger correlation between $\pi$ and $r$ in genomic regions that have not undergone dramatic

192 changes in the recombination rate.

193 Using an alignment of genomes from closely related species, Thybert et al. (2018) distinguished

194 chromosomes in the M. musculus genome that have or have not undergone dramatic

195 rearrangements in the last 5 million years from those that have not. We re-analysed data from

196 Kartje et al. (2020) and found that the correlation between $\pi$ and $r$ is stronger and more

197 significant on chromosomes that have not undergone largescale rearrangements in the last 3-5

198 million years (Table 1) for M. m. domesticus individuals from France and Germany. This pattern

199 holds when looking at analysis windows of $5 \mathrm{Kbp}$ and $1 \mathrm{Mbp}$ (Table 1). No substantial

200 correlations were found for mice from Gough Island in any comparison. M. m. domesticus are

201 believed to have colonised Gough Island in the 19th century and to have experienced a severe

202 population bottleneck (Gray et al. 2014), a demographic history that could have further

203 obscured the correlation between nucleotide diversity and recombination rate in that

204 population. 
Table 1. The correlation between nucleotide diversity $(\pi)$ and recombination rate $(r)$ for three populations of house mice (Mus musculus domesticus) calculated from all autosomes, conserved chromosomes that exhibit no syntenic breaks between $M$. musculus and $M$. pahari and chromosomes that experienced large scale rearrangements as identified by Thybert et al. (2018). Correlations with $p$-values less than 0.01 are highlighted in bold text.

\begin{tabular}{|c|c|c|c|c|c|c|c|}
\hline \multirow{2}{*}{$\begin{array}{l}\text { Window } \\
\text { Size }\end{array}$} & \multirow{2}{*}{ Population } & \multicolumn{2}{|c|}{ Whole Genome } & \multicolumn{2}{|c|}{ Conserved Chromosomes } & \multicolumn{2}{|c|}{ Rearranged Chromosomes } \\
\hline & & Spearman's $\rho$ & $p$-value & Spearman's $\rho$ & $p$-value & Spearman's $\rho$ & $p$-value \\
\hline $5 \mathrm{Kbp}$ & Gough Island & 0.00767 & $4.28 \times 10^{-5}$ & 0.00880 & 0.0102 & 0.00486 & 0.0302 \\
\hline $5 \mathrm{Kbp}$ & France & 0.00408 & 0.0295 & 0.0403 & $6.10 \times 10^{-32}$ & -0.0107 & $1.76 \times 10^{-6}$ \\
\hline $5 \mathrm{Kbp}$ & Germany & 0.00752 & $6.05 \times 10^{-5}$ & 0.0152 & $9.63 \times 10^{-6}$ & 0.00386 & 0.0849 \\
\hline $1 \mathrm{Mbp}$ & Gough Island & 0.0536 & 0.00946 & 0.0588 & 0.124 & 0.0437 & 0.0748 \\
\hline $1 \mathrm{Mbp}$ & France & 0.0450 & 0.0294 & 0.135 & 0.000400 & 0.00999 & 0.684 \\
\hline $1 \mathrm{Mbp}$ & Germany & 0.0535 & 0.00953 & 0.0775 & 0.0428 & 0.0426 & 0.0828 \\
\hline
\end{tabular}

\section{Discussion}

213 Evolution of the recombination rate will influence the effects of selection at linked sites (e.g.

214 BGS and selective sweeps) and thus influence patterns of genetic variability. Estimates of the

215 recombination rate made from contemporary populations may not adequately predict genetic

216 variability up to $2 N_{e}$ generations following evolution of the recombination rate landscape

217 (Figure 1, 2). Our re-analysis of the Kartje et al. (2020) data suggests that mice are still within

218 the lag period after evolution of the recombination rate, such that $\pi$ in $M . m$. domesticus does

219 not fully reflect contemporary recombination rates in Mus musculus. In contrast, the ancestors

220 of Heliconius butterflies also underwent large-scale karyotype evolution, but gross patterns of $\pi$

221 versus chromosome length in those species suggest that patterns of variation have largely re-

222 equilibrated after changes in $r$ (Cicconardi et al. 2021). 
223 While our re-analysis of the data from Kartje et al. (2020) suggests that recombination rate

224 evolution in the ancestors of mice obscures the evidence for natural selection across the

225 genome, the overall correlations between $\pi$ and $r$ were still fairly weak on the conserved

226 chromosomes (Table 1). The largest rank correlation coefficient we found was 0.135 for the

227 sample of M. m. domesticus from France (1Mbp windows; Table 1). By contrast, Spearman's

228 rank correlation between nucleotide diversity and recombination rate in humans has been

229 reported to be 0.219 for $400 \mathrm{Kbp}$ analysis windows (Cai et al. 2009). The variance in

230 recombination rates across the $M$. musculus genome is less than a half that which has been

231 reported for humans (Jensen-Seaman et al. 2004), so perhaps the effects of BGS across the

232 genome are more homogenous in M. musculus than they are in humans, contributing to the

233 weak correlations between $\pi$ and $r$ shown in Table 1. Beyond the pulse of karyotype evolution

234 reported by Thybert et al. (2018), there is clear evidence of recent and likely ongoing evolution

235 of the recombination rate in M. musculus (see Introduction), which may further obscure

236 genome-wide evidence for the effects of natural selection. For example, there is strong

237 evidence that the landscape of recombination hotspots in the M. musculus genome has evolved

238 rapidly among sub-species and populations (Smagulova et al. 2015). Our simulations suggest

239 that even a single change to the locations of hotspots can substantially weaken the correlation

240 between $\pi$ and $r$ (Figure 2, S6). Of course, there are reasons why species may not exhibit a

241 strong positive correlation between $\pi$ and $r$ that have nothing to do with recombination rate

242 evolution (Cutter and Payseur 2013). For example, wild and domesticated rice (Oryza spp.)

243 exhibit negative correlations between $\pi$ and $r$, but in those species there is a strong positive

244 correlation between the density of functional sites (i.e. sites subject to purifying selection) and 
245 the recombination rate (Flowers et al. 2011). In such a case, the effects of BGS are primarily

246 occurring in regions of high recombination.

247 This short paper should add to the growing appreciation of recombination as an evolutionarily

248 labile trait. As pointed out by Comeron (2017) and Smukowski Heil et al. (2015), information on

249 recombination rates in outgroup species is an important covariate when performing population

250 genomic analyses. In some lineages, recombination rates may evolve very slowly. Birds, for

251 example, have highly conserved karyotypes and in some cases highly conserved recombination

252 landscapes (Damas et al. 2018; Singhal et al. 2015). Evolution of the recombination rate is

253 another of the many possible reasons why one might not be able to adequately identify the

254 effects of BGS (or natural selection more broadly) from population genomic data (See reviews

255 by Cutter and Payseur 2013 and Comeron 2017), but conservation of recombination landscapes

256 will likely make comparative population genomics more straightforward.

\section{Methods}

\section{Model}

259 Background selection has been modelled as the reduction in effective population size $\left(N_{e}\right)$ at a

260 neutral site due to the removal of linked deleterious variants. The effects of background

261 selection are often expressed as $B=\frac{N_{e}}{N_{0}}$, where $N_{e}$ is the effective population size and $N_{0}$ is the

262 expected population size under strict neutrality. In a non-recombining genome, $B$ is

263 proportional to the ratio of the deleterious mutation rate to the strength of selection acting on 
264 harmful mutations (Charlesworth et al. 1993). For a neutral site present on a recombining

265 chromosome, the effects of background selection depend on the density of functional sites (i.e.

266 those that can mutate to deleterious alleles), the strength of selection at functional sites, the

267 mutation rate at functional sites and the recombination rate between the neutral site and the

268 functional sites (Hudson and Kaplan 1995; Nordborg et al. 1996; Nordborg 1997). For a neutral

269 locus $v$ linked to $x$ functional sites, the reduction in $N_{e}$ has been described with the following

270 equation:

$$
B_{v}=\frac{N_{e}}{N_{0}}=\exp \left[-\sum_{x} \frac{u_{x}}{t\left(1+(1-t) r_{x, v} / t\right)^{2}}\right]
$$

272 where $u_{x}$ is the deleterious mutation rate at functional site $x, t$ is the heterozygous fitness

273 effect of a deleterious mutation (i.e. $0.5 \mathrm{~s}$ in the case of semi-dominance) and $r_{x, v}$ is the

274 recombination map distance between the neutral locus and functional site $x$. In the above

275 equation, deleterious mutations have fixed effects, but it is straightforward to incorporate a

276 distribution of fitness effects (Nordborg et al. 1996). The above equation holds when selection

277 is sufficiently strong such that random drift does not overwhelm selection $\left(N_{e} s>1\right)$ (Good et

278 al. 2014).

280 Simulations

281 We simulated BGS under recombination rate evolution using two types of simulations in SLiM

282 v3.2 (Haller and Messer 2019). We simulated diploid populations of $N_{e}=5,000$ individuals. In all 
283 cases, we scaled mutation, recombination and the strength of selection to approximate

284 evolution in a large population.

286 The first set of simulations was designed to examine how long it takes for patterns of neutral

287 diversity under BGS to equilibrate after the recombination rate evolves. In these simulations,

288 the genome was $25 \mathrm{Kbp}$ long with a $5 \mathrm{Kbp}$ functional element in the centre. Mutations occurred

289 in the functional element at rate $\mu=5 \times 10^{-7}$ and had semi-dominant fitness effects with a

290 fixed selection coefficient of $s=-0.01$. We also simulated cases with varying fitness effects

291 using a gamma distribution with mean $(\bar{S})$ of -0.1 and a shape parameter of 0.1 . Recombination

292 occurred at a uniform rate of $r=5 \times 10^{-7}$ across the chromosome. After 80,000 generations

293 (16Ne generations), we simulated an instantaneous change in the recombination rate,

294 multiplying $r$ by $\lambda$, giving $r=\lambda 5 \times 10^{-7}$. We simulated cases with $\lambda=0.1,1.0$ and 10.0.

295 Simulated populations were sampled every 2,500 generations after the recombination rate

296 changed and we performed 200 replicates for each set of parameters tested. Note that these

297 simulations were not designed to be particularly realistic, but to provide clear cut patterns to

298 test the theoretical predictions.

300 The second set of simulations was designed to examine how patterns of $\pi$ versus $r$ varied over

301 time when recombination rates evolved at fine and/or broad scales. For these simulations, we

302 modelled chromosomes that were $10 \mathrm{Mbp}$ long. Neutral mutations occurred at random across

303 the length of the sequence at a rate of $5 \times 10^{-7}$ (such that expected nucleotide diversity was

304 0.01). Deleterious mutations occurred at random across the length of the sequence at a rate of 
$3055 \times 10^{-8}$ with semi-dominant fitness effects drawn from a gamma distribution with a mean $(\bar{S})$

306 of -0.1 and a shape parameter of 0.1 . The deleterious mutation rate was chosen so that $10 \%$ of

307 the genome was subject to purifying selection. Populations evolved under background selection

308 for 80,000 generations (i.e. $16 N_{e}$ generation). In generation 80,000 there was instantaneous

309 evolution of the recombination landscape after which we recorded the tree-sequence of the

310 population every 5,000 generations for a further 40,000 generations. We incorporated two

311 models of recombination rate variation and evolution of the recombination map:

312 - We modelled recombination rate evolution at broad scales by rearrangement of the

313 recombination landscape. Recombination rates vary across the genome (Stapley et al.

314 2017). For example, recombination rates vary by a factor of 3 across chromosome 1 in

315 mice. In these simulations, recombination varied from $r=2.08 \times 10^{-7}$ to $r=$

$3166.24 \times 10^{-7}$ across the simulated chromosome (Figure S4A). When the recombination

317 landscape evolved, we reversed the order of recombination rates across the genome

$318 \quad$ (Figure S4A).

319 - We modelled evolution of the recombination map by the movement of hotspots.

320 Recombination occurred at a uniform rate of $r=6 \times 10^{-8}$ except in $5 \mathrm{Kbp}$ hotspots

321 where it occurred at a rate of $r=6 \times 10^{-6}$. At the beginning of a simulation, a Poisson

322 number of hotspots was sampled with an expectation of 120 . Hotspots were placed at

323 random across the simulated chromosome. When the recombination landscape evolved,

324 we resampled the locations of hotspots (Figure S4B). 
325 In both cases, rates were chosen such that the total map length was similar to one that

326 recombined at a constant rate of $4 N_{e} r=0.008$, the value reported for wild mice (Booker et al.

327 2017). For both models of recombination rate map evolution, we performed 20 simulation

328 replicates, giving a total of $200 \mathrm{Mbp}$ worth of simulated data, similar to the length of

329 chromosome 1 in mice.

331 For all simulations, we used the tree sequence recording option in SLiM and neutral mutations

332 were added to the resulting tree-sequences at a rate of $5 \times 10^{-7}$ using PySLiM and msprime

333 (Haller et al. 2019; Kelleher et al. 2016). Nucleotide diversity $(\pi)$ was calculated in windows of

334 varying size using sci-kit-allel. We used the program PyRho (Spence and Song 2019) to estimate

335 recombination rates from samples of 10 diploid individuals from 20 replicate simulations.

336 Spearman's $\rho$ between $\pi$ and $r$ was calculated using R. All figures were made using ggplot2. All

337 simulation scripts and analysis and plotting scripts are deposited at

338 https://github.com/TBooker/BGS_RecombinationRateEvolution.

\section{Acknowledgements}

340 Thanks to Nadia Singh and Judith Mank for the invitation to present this work at vSMBE 2021. 341 Thanks to Michael Whitlock, Brian Charlesworth and Mikey Kartje for helpful discussions. TRB

342 was funded by a Bioinformatics Fellowship awarded by the Biodiversity Research Centre at the 343 University of British Columbia. BAP was supported by NIH R35 GM139412. 


\section{References}

Auton, Adam, et al. "Genetic recombination is targeted towards gene promoter regions in dogs." PLoS genetics 9.12 (2013): e1003984.

348 Baker, Zachary, Molly Schumer, Yuki Haba, Lisa Bashkirova, Chris Holland, Gil G. Rosenthal, and Molly Przeworski. 2017. "Repeated losses of PRDM9-directed recombination despite the conservation of PRDM9 across vertebrates." eLife 6

Baudat, F, J Buard, C Grey, A Fledel-Alon, C Ober, M Przeworski, G Coop, and B de Massy. 2010. "PRDM9 Is a Major Determinant of Meiotic Recombination Hotspots in Humans and Mice."

353 Science 327 (5967): 836-40.

354 Booker, T R, R W Ness, and P D Keightley. 2017. "The recombination landscape in wild house 355 mice inferred using population genomic data." Genetics 207 (1): 297-309.

356 Brick, Kevin, et al. "Genetic recombination is directed away from functional genomic elements 357 in mice." Nature 485.7400 (2012): 642-645.

Cai, James J., et al. "Pervasive hitchhiking at coding and regulatory sites in humans." PLoS genetics 5.1 (2009): e1000336.

Campos, J L, L Zhao, and B Charlesworth. 2017. "Estimating the parameters of background selection and selective sweeps in Drosophila in the presence of gene conversion." Proceedings of the National Academy of Sciences 114 (24): E4762-E4771.

365 Charlesworth, B. 2009. "Fundamental concepts in genetics: effective population size and 366 patterns of molecular evolution and variation." Nat Rev Genet 10 (3): 195-205.

367 Charlesworth, B, M T Morgan, and D Charlesworth. 1993. "The effect of deleterious mutations 368 on neutral molecular variation." Genetics 134: 1289-1303.

369 Chmátal, Lukáš, Sofia I. Gabriel, George P. Mitsainas, Jessica Martínez-Vargas, Jacint Ventura, 370 Jeremy B. Searle, Richard M. Schultz, and Michael A. Lampson. 2014. "Centromere Strength

371 Provides the Cell Biological Basis for Meiotic Drive and Karyotype Evolution in Mice." Current

372 Biology 24 (19): 2295-2300.

373 Cicconardi, Francesco, James J Lewis, Simon H Martin, Robert D Reed, Charles G Danko, and 374 Stephen H Montgomery. 2021. "Chromosome Fusion Affects Genetic Diversity and Evolutionary 375 Turnover of Functional Loci but Consistently Depends on Chromosome Size." Molecular Biology 376 and Evolution 38 (10): 4449-62. 
377 Comeron, Josep M. 2017. "Background selection as null hypothesis in population genomics:

378 insights and challenges from Drosophila studies." Philos Trans R Soc Lond B Biol Sci 372 (1736).

379 Cox, Allison, Cheryl L. Ackert-Bicknell, Beth L. Dumont, Yueming Ding, Jordana Tzenova Bell, 380 Gudrun A. Brockmann, Jon E. Wergedal, et al. 2009. "A New Standard Genetic Map for the 381 Laboratory Mouse." Genetics 182 (4): 1335-44.

382 Cutter, A D, and B A Payseur. 2013. "Genomic signatures of selection at linked sites: unifying 383 the disparity among species." Nat Rev Genet 14 (4): 262-74.

384 Cvijović, Ivana, Benjamin H Good, and Michael M Desai. 2018. "The Effect of Strong Purifying 385 Selection on Genetic Diversity." Genetics 209 (4): 1235-78.

Damas, Joana, Jaebum Kim, Marta Farré, Darren K. Griffin, and Denis M. Larkin. 2018. "Reconstruction of Avian Ancestral Karyotypes Reveals Differences in the Evolutionary History of Macro- and Microchromosomes." Genome Biology 19 (1): 155. 1895-7.

392 Dumont, Beth L., and Bret A. Payseur. "Evolution of the genomic recombination rate in murid 393 rodents." Genetics 187.3 (2011): 643-657.

394 Dumont, Beth L., et al. "Extensive recombination rate variation in the house mouse species 395 complex inferred from genetic linkage maps." Genome research 21.1 (2011): 114-125.

396 Dumont, Beth L. "Variation and evolution of the meiotic requirement for crossing over in 397 mammals." Genetics 205.1 (2017): 155-168.

398 Eyre-Walker, Adam, and Peter D. Keightley. "The distribution of fitness effects of new 399 mutations." Nature Reviews Genetics 8.8 (2007): 610-618.

400 Flowers, Jonathan M., Jeanmaire Molina, Samara Rubinstein, Pu Huang, Barbara A. Schaal, and 401 Michael D. Purugganan. 2011. "Natural Selection in Gene-Dense Regions Shapes the Genomic 402 Pattern of Polymorphism in Wild and Domesticated Rice." Molecular Biology and Evolution 29 403 (2): 675-87.

404 Good, Benjamin H., Aleksandra M. Walczak, Richard A. Neher, and Michael M. Desai. 2014. 405 "Genetic Diversity in the Interference Selection Limit." PLoS Genetics 10 (3).

406 Gray, Melissa M., Daniel Wegmann, Ryan J. Haasl, Michael A. White, Sofia I. Gabriel, Jeremy B. 407 Searle, Richard J. Cuthbert, Peter G. Ryan, and Bret A. Payseur. 2014. “Demographic History of a 
408 Recent Invasion of House Mice on the Isolated Island of Gough." Molecular Ecology 23 (8):

409 1923-39.

410 Haller, Benjamin C, Jared Galloway, Jerome Kelleher, Philipp W Messer, and Peter L Ralph. 411 2019. "Tree-sequence recording in SLiM opens new horizons for forward-time simulation of

412 whole genomes." Molecular Ecology Resources 19 (2): 552-66.

413 Haller, Benjamin C, and Philipp W Messer. 2019. "SLiM 3: Forward Genetic Simulations Beyond 414 the Wright-Fisher Model." Molecular Biology and Evolution 36 (3): 632-37.

415 Hudson, R R, and N L Kaplan. 1995. “Deleterious background selection with recombination." 416 Journal Article. Genetics 141: 1605-17.

417 Jensen-Seaman, Michael I., et al. "Comparative recombination rates in the rat, mouse, and 418 human genomes." Genome research 14.4 (2004): 528-538.

419 Johri, Parul, Brian Charlesworth, and Jeffrey D. Jensen. 2020. "Toward an evolutionarily 420 appropriate null model: Jointly inferring demography and purifying selection." Genetics 215 (1): 421 173-92.

422 Kartje, Michael E., Peicheng Jing, and Bret A. Payseur. 2020. "Weak Correlation between 423 Nucleotide Variation and Recombination Rate across the House Mouse Genome." Genome 424 Biology and Evolution 12 (4): 293-99.

425 Kelleher, Jerome, Alison M Etheridge, and Gilean McVean. 2016. "Efficient Coalescent 426 Simulation and Genealogical Analysis for Large Sample Sizes." PLOS Computational Biology 12 427 (5): e1004842.

428 Leffler, E M, K Bullaughey, D R Matute, W K Meyer, L Segurel, A Venkat, P Andolfatto, and M 429 Przeworski. 2012. "Revisiting an old riddle: what determines genetic diversity levels within 430 species?” PLoS Biology 10 (9): e1001388.

431 Nordborg, M. 1997. "Structured coalescent processes on different time scales." Genetics 146 432 (4): 1501-14.

433 Nordborg, M, B Charlesworth, and D Charlesworth. 1996. "The effect of recombination on 434 background selection." Journal Article. Genetical Research 67: 159-74.

435 Oliver, P L, L Goodstadt, J J Bayes, Z Birtle, K C Roach, N Phadnis, S A Beatson, G Lunter, H S 436 Malik, and C P Ponting. 2009. "Accelerated evolution of the Prdm9 speciation gene across 437 diverse metazoan taxa." PLoS Genetics 5 (12): e1000753. 
Paigen, K, J P Szatkiewicz, K Sawyer, N Leahy, E D Parvanov, S H Ng, J H Graber, K W Broman, and P M Petkov. 2008. "The recombinational anatomy of a mouse chromosome." PLoS Genetics 4 (7): e1000119.

441 Pardo-Manuel de Villena, Fernando, and Carmen Sapienza. "Recombination is proportional to

442 the number of chromosome arms in mammals." Mammalian Genome 12.4 (2001): 318-322.

443 Peterson, April L., and Bret A. Payseur. "Sex-specific variation in the genome-wide

444 recombination rate." Genetics 217.1 (2021): 1-11.

445 Pool, J E, and R Nielsen. 2009. “Correction for Pool and Nielsen (2007)." Evolution 63 (6): 16714461.

447 Segura, Joana, et al. "Evolution of recombination in eutherian mammals: insights into 448 mechanisms that affect recombination rates and crossover interference." Proceedings of the 449 Royal Society B: Biological Sciences 280.1771 (2013): 20131945.

450 Singhal, Sonal, Ellen M. Leffler, Keerthi Sannareddy, Isaac Turner, Oliver Venn, Daniel M. 451 Hooper, Alva I. Strand, et al. 2015. "Stable recombination hotspots in birds." Journal Article. 452 Science 350 (6263): 928-32.

453 Smagulova, F, K Brick, P Yongmei, R D Camerini-Otero, and G V Petukhova. 2016. "The 454 evolutionary turnover of recombination hotspots contributes to speciation in mice." Journal 455 Article. Genes \& Development 30: 277-80.

456 Smukowski Heil, C S, C Ellison, M Dubin, and M A Noor. 2015. "Recombining without Hotspots: 457 A Comprehensive Evolutionary Portrait of Recombination in Two Closely Related Species of 458 Drosophila." Genome Biology and Evolution 7 (10): 2829-42.

459 Spence, Jeffrey P., and Yun S. Song. 2019. "Inference and analysis of population-specific fine460 scale recombination maps across 26 diverse human populations." Science Advances 5 (10).

461 Stapley, Jessica, Philine G. D. Feulner, Susan E. Johnston, Anna W. Santure, and Carole M. 462 Smadja. 2017. "Variation in Recombination Frequency and Distribution Across Eukaryotes: 463 Patterns and Processes." Philosophical Transactions of the Royal Society B: Biological Sciences 464372 (1736): 20160455.

465 Sun, Hequan, Beth A. Rowan, Pádraic J. Flood, Ronny Brandt, Janina Fuss, Angela M. Hancock, 466 Richard W. Michelmore, Bruno Huettel, and Korbinian Schneeberger. 2019. "Linked-read 467 sequencing of gametes allows efficient genome-wide analysis of meiotic recombination." 468 Nature Communications 10 (1): 1-9. 
469 Thybert, David, Maša Roller, Fábio C. P. Navarro, lan Fiddes, Ian Streeter, Christine Feig, David 470 Martin-Galvez, et al. 2018. "Repeat associated mechanisms of genome evolution and function 471 revealed by the Mus caroli and Mus pahari genomes." Genome Research 28 (4): 448-59.

472 Vara, Covadonga, Andreu Paytuví-Gallart, Yasmina Cuartero, Lucía Álvarez-González, Laia 473 Marín-Gual, Francisca Garcia, Beatriu Florit-Sabater, et al. 2021. "The impact of chromosomal 474 fusions on 3D genome folding and recombination in the germ line." Nature Communications 4752021 12:1 $12(1): 1-17$.

476 Wang, Richard J., et al. "Recombination rate variation in mice from an isolated 477 island." Molecular ecology 26.2 (2017): 457-470. 


\section{Supplementary Material}

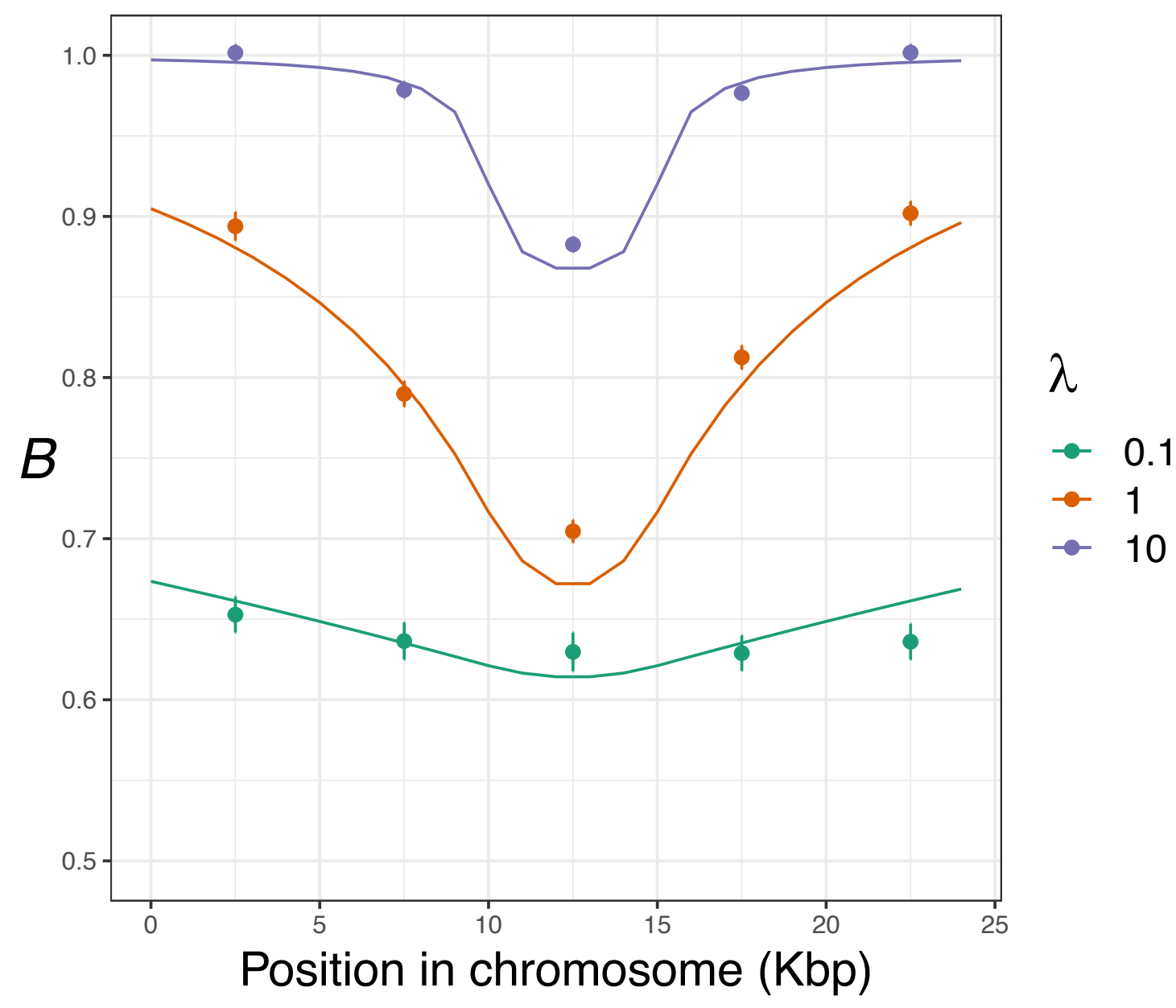

481 Figure S1. The effects of background selection across simulated chromosomes. B was calculated 482 for simulated data by comparing observed $\pi$ to the neutral expectation of $4 N_{e} \mu=0.01$. The 483 lines show the theoretical expectation calculated using formulae from Nordborg et al (1996). 


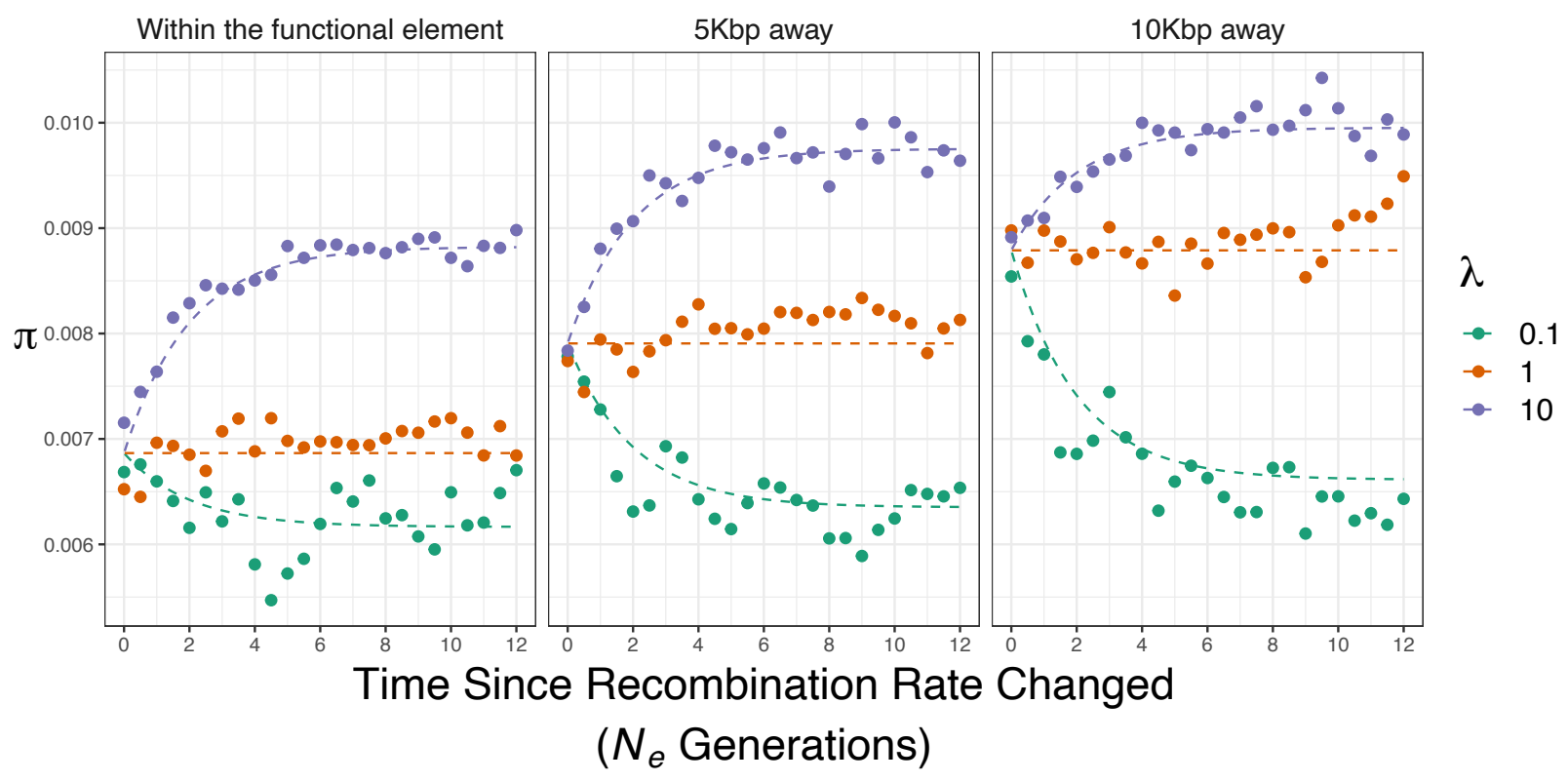

486 Figure S2. The effects of background selection across simulated chromosomes. B was calculated for simulated data by comparing observed $\pi$ to the neutral expectation of $4 N_{e} \mu=0.01$. The lines show the theoretical expectation calculated using Equation 1 and formulae from Nordborg et al (1996). The labels on the top of each panel indicate the location in the simulated data

490 being analysed. 


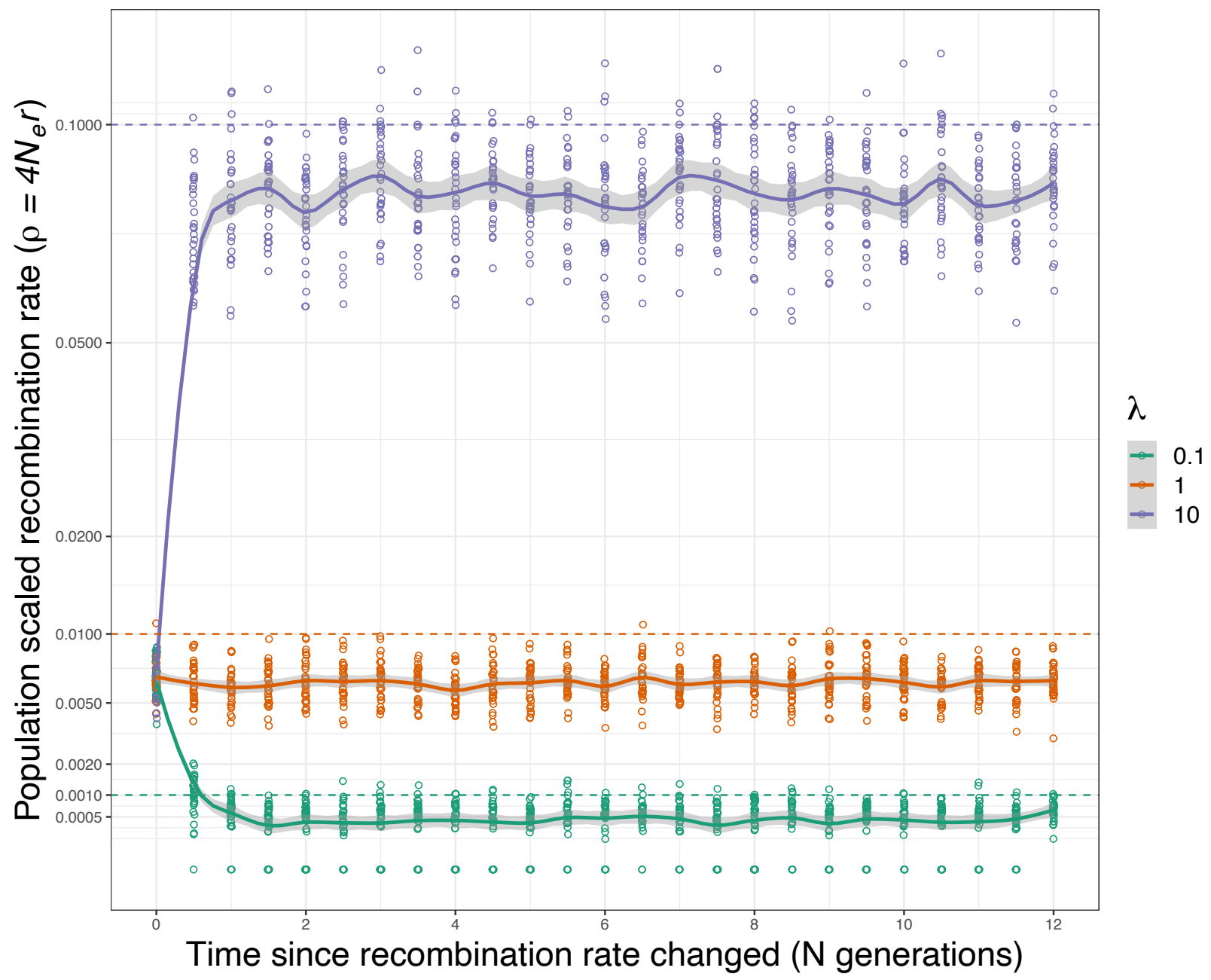

492 Figure S3. Recombination rates inferred using PyRho after an instantaneous change in the recombination rate. Dashed horizontal lines indicate the true recombination rate for the three cases. Smoothed lines with shaded ribbon indicate the fit and error of a LOESS regression. of $\lambda$. 
A

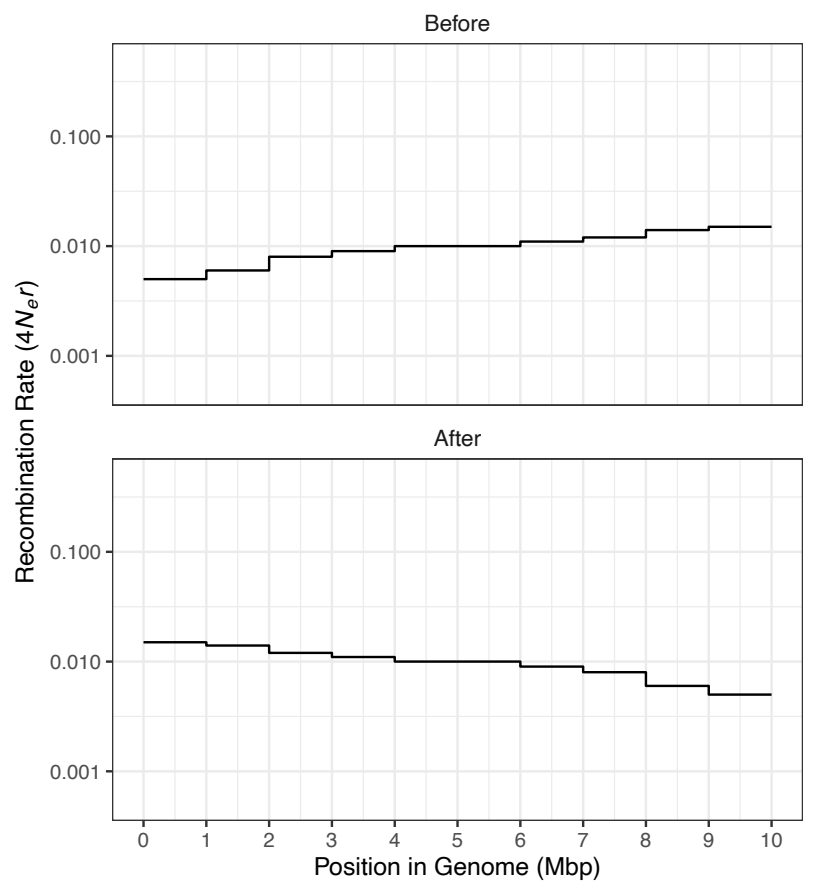

B

Recombination Hotspots

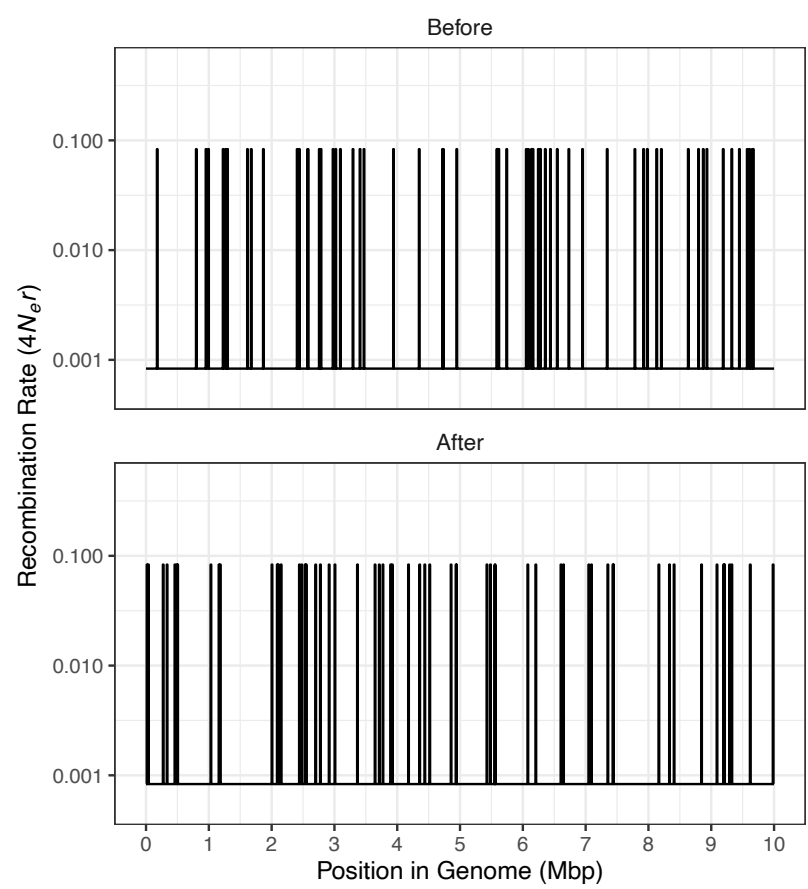

498 Figure S4. The recombination rate maps used in the simulations modelling BGS across the genome. The upper and lower panels show the recombination rate landscape before and after

500 it evolved in simulations, respectively. A) Evolution of the recombination rate at the Mbp scale.

501 B) Evolution of the recombination rate at the scale of recombination hotspots. 
A
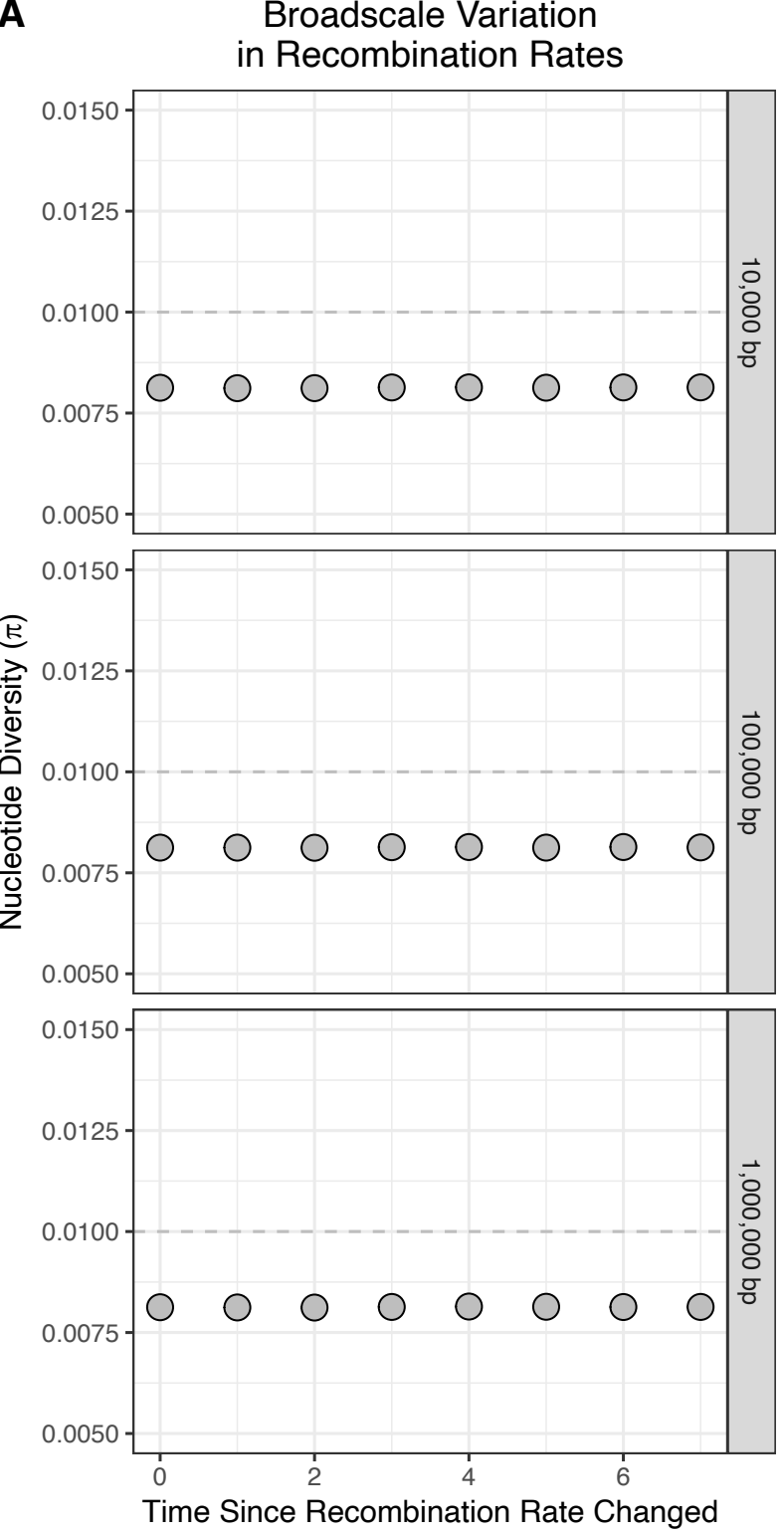

$\left(N_{e}\right.$ Generations)
B

Recombination

Hotspots
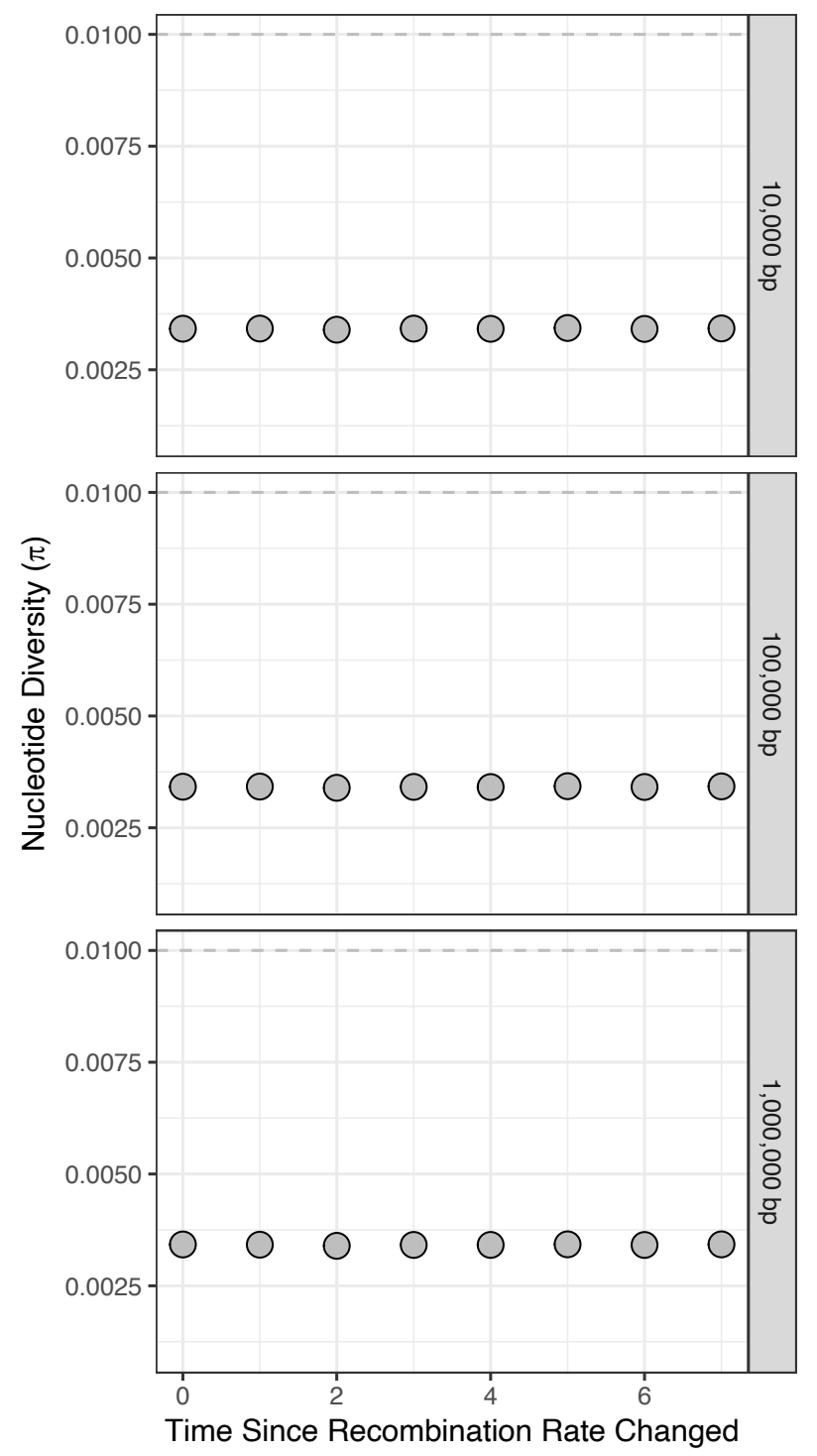

$\left(N_{e}\right.$ Generations)

503 Figure S5. Nucleotide diversity $(\pi)$ over time after evolution of the recombination landscape.

504 Panel A) shows results for the model of broadscale recombination rate evolution. Panel B)

505 shows the results for the model of recombination hotspot evolution. The dashed horizontal

506 grey line indicates the null expectation of $4 N_{e} \mu=0.01$. In both panels, the text in the grey strips 507 to the right of each cell indicates the size of analysis windows used. 


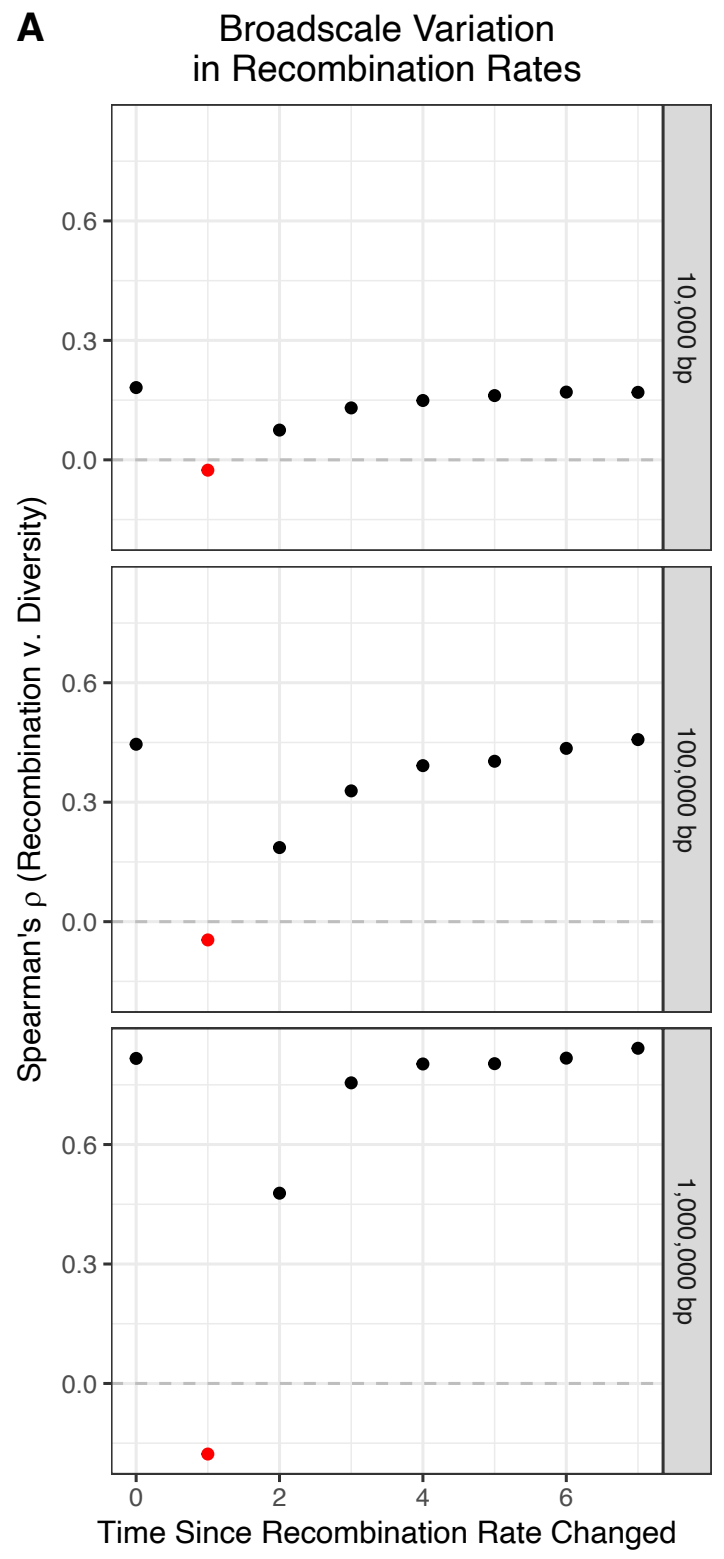

509

510

511

512

513

514
B
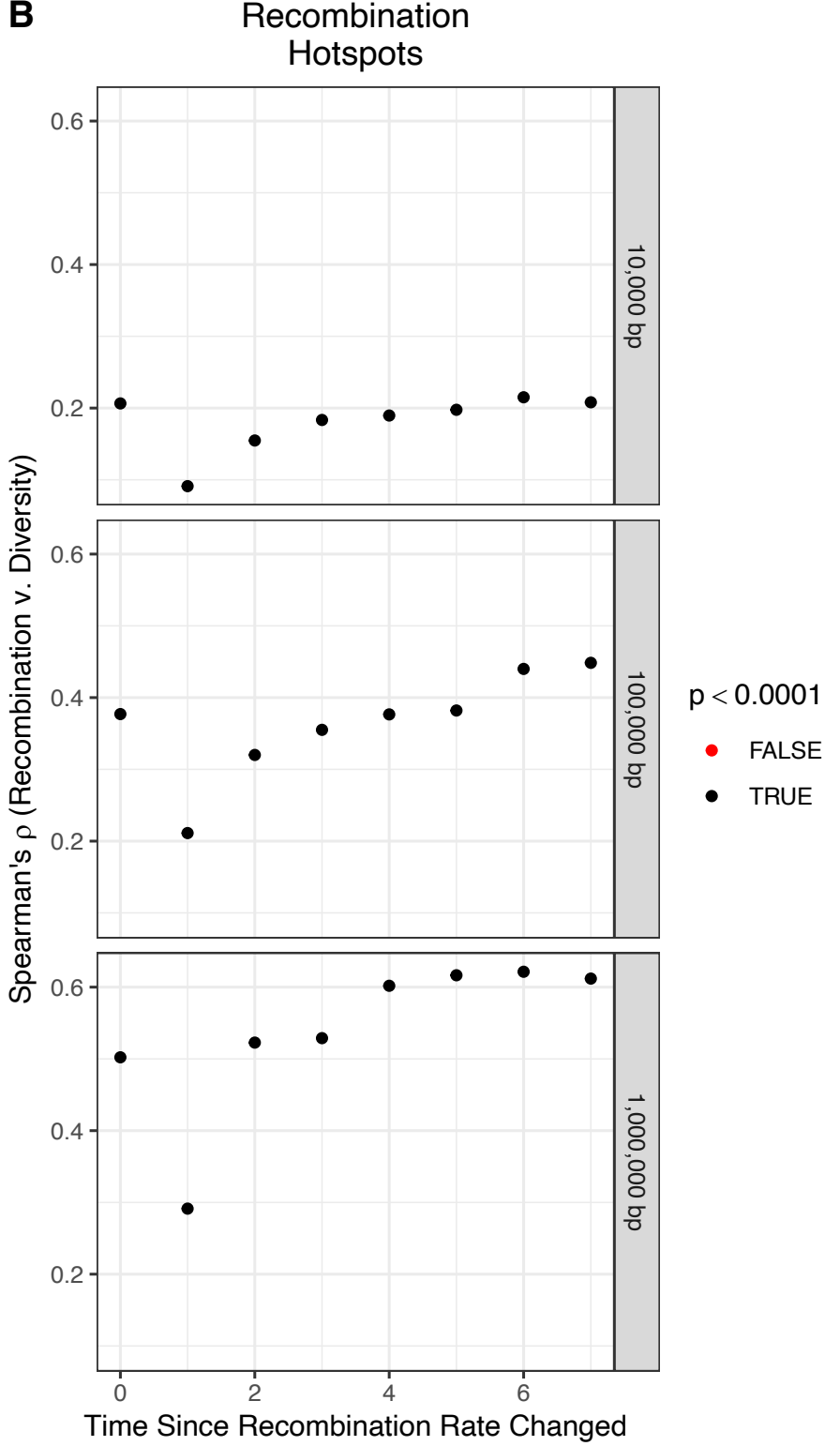

( $N_{e}$ Generations) 